\title{
New features and improved uncertainty analysis in the NEA nuclear data sensitivity tool (NDaST)
}

\author{
J. Dyrda, N. Soppera, I. Hill, M. Bossant, and J. Gulliford \\ OECD-NEA, 46 quai Alphonse Le Gallo, 92100 Boulogne-Billancourt, France
}

\begin{abstract}
Following the release and initial testing period of the NEA's Nuclear Data Sensitivity Tool [1], new features have been designed and implemented in order to expand its uncertainty analysis capabilities. The aim is to provide a free online tool for integral benchmark testing, that is both efficient and comprehensive, meeting the needs of the nuclear data and benchmark testing communities. New features include access to P1 sensitivities for neutron scattering angular distribution [2] and constrained Chi sensitivities for the prompt fission neutron energy sampling. Both of these are compatible with covariance data accessed via the JANIS nuclear data software, enabling propagation of the resultant uncertainties in $k_{\text {eff }}$ to a large series of integral experiment benchmarks. These capabilities are available using a number of different covariance libraries e.g., ENDF/B, JEFF, JENDL and TENDL, allowing comparison of the broad range of results it is possible to obtain. The IRPhE database of reactor physics measurements is now also accessible within the tool in addition to the criticality benchmarks from ICSBEP. Other improvements include the ability to determine and visualise the energy dependence of a given calculated result in order to better identify specific regions of importance or high uncertainty contribution. Sorting and statistical analysis of the selected benchmark suite is now also provided. Examples of the plots generated by the software are included to illustrate such capabilities. Finally, a number of analytical expressions, for example Maxwellian and Watt fission spectra will be included. This will allow the analyst to determine the impact of varying such distributions within the data evaluation, either through adjustment of parameters within the expressions, or by comparison to a more general probability distribution fitted to measured data. The impact of such changes is verified through calculations which are compared to a 'direct' measurement found by adjustment of the original ENDF format file.
\end{abstract}

\section{Revised input and output features}

Several new features revising the overall "look" of the NDaST software have been developed for the 2016 release version. Here we describe and illustrate the most important of these new-look input and output features:

In addition to the Database for the International Handbook of Evaluated Criticality Safety Benchmark Experiments (DICE), the new version allows access to benchmarks from the International Reactor Physics Handbook Database and Analysis Tool (IDAT). This uses the same familiar search interface as the main IDAT version, with some bespoke modifications. The data accessible is currently limited to criticality measurements, as these are the only data to which sensitivity profiles are associated within the NEA online database. Users' own benchmarks and sensitivities for other integral parameters may still be input via the 'New Benchmark' feature. Sorting of output figures by individual and common criteria (for instance evaluation ID, spectrum, calculation code etc.) now takes into account DICE and IDAT properties in a single plot.

Similarly to the DICE and IDAT software tool plots, NDaST now features 'tool-tips'; these floating windows appear when the cursor rests on a bar within a plot, giving the underlying quantitative values. This will now allow users to quickly access data on the number of points constituting each 'grouping' and mean and standard deviation values for $\Delta$ keff, original and perturbed keff $\mathrm{C} / \mathrm{E}$ values and the propagated nuclear data uncertainty.

There have also been several fundamental improvements to the output window presentation. Firstly, in addition to the $\Delta k_{\text {eff }}$ and data variance, the output table now shows additional columns for the (mean) original and perturbed $k_{\text {eff }} \mathrm{C} / \mathrm{E}$ values of each benchmark. The new 'detail popup' format for each benchmark now breaks these down for each code / library combination, as well as the perturbation and uncertainty breakdown into nuclidereaction pairs. Finally, the filter tree now added to the side of the plot allows nuclide-reaction pairs and code / library combination results to be included or excluded from the display with automatic live update of the resulting plot and table (see, for example, Fig. 1 and Fig. 2).

\section{Automated JANIS computations}

In order to more easily facilitate the input of perturbation series to NDaST, an automated link has been introduced to the JANIS nuclear data viewing software. From a search dialogue within the 'Perturbations' panel of NDaST, the user may search their public / private JANIS databases for a given nuclide and reaction combination. Selection of two nuclear data evaluations returned from this search as numerator and denominator prompts the software to generate the relevant perturbation ratios. This ratio is 


\begin{tabular}{|c|c|c|c|c|c|}
\hline NDaST & & & & & 回 \\
\hline \multicolumn{6}{|l|}{ File } \\
\hline Filter & Benchmark & $\Delta$ Keff/Keff & Keff $C / E$ original & Keff $C / E$ perturbed & Data Variance \\
\hline 1) Filter & PMF001-001 (Detaile... & KENO ENDF $/ B$-VII. $0 / \ldots 10$ & 1.00001 & 1.00001 & $1.92066478 \mathrm{e}-5$ \\
\hline - Nudides / Reactions & PMF002-001 & KENO ENDF/B-VII.O / ... & 1 & 1 & $1.38853928 \mathrm{e}-5$ \\
\hline V & PMF006-001 & KENO ENDF/B-VII. $0 / \ldots 0$ & 1 & $9.99895426 \mathrm{e}-1$ & $1.46876237 \mathrm{e}-5$ \\
\hline (t) & PMI002-001 & KENO ENDF/B-VII.O / ... & 1.01327389 & 1.01327389 & $4.26859905 e^{-}-5$ \\
\hline t) - 02335 & HMI001-001 & KENO ENDF/B-VII. $0 / \ldots 0$ & 1.0034116 & 1.00481944 & 0 \\
\hline Pu239 & MMI003-001 & KENO ENDF/B-VII. $0 / \ldots 0$ & 1.03755966 & 1.03755705 & $4.23083286 \mathrm{e}^{-5}$ \\
\hline (4) Pu239 & MML004-001 & KENO ENDF/B-VII.O / . 0 & 1.0249052 & 1.02482391 & $1.84266684 \mathrm{e}-5$ \\
\hline Sensitivities calculations & MCF001-001 & KENO ENDF/B-VII. $0 / \ldots 0$ & 1.013582 & 1.01347515 & $1.53765113 \mathrm{e}-5$ \\
\hline
\end{tabular}

Figure 1. Output table with tree filter.

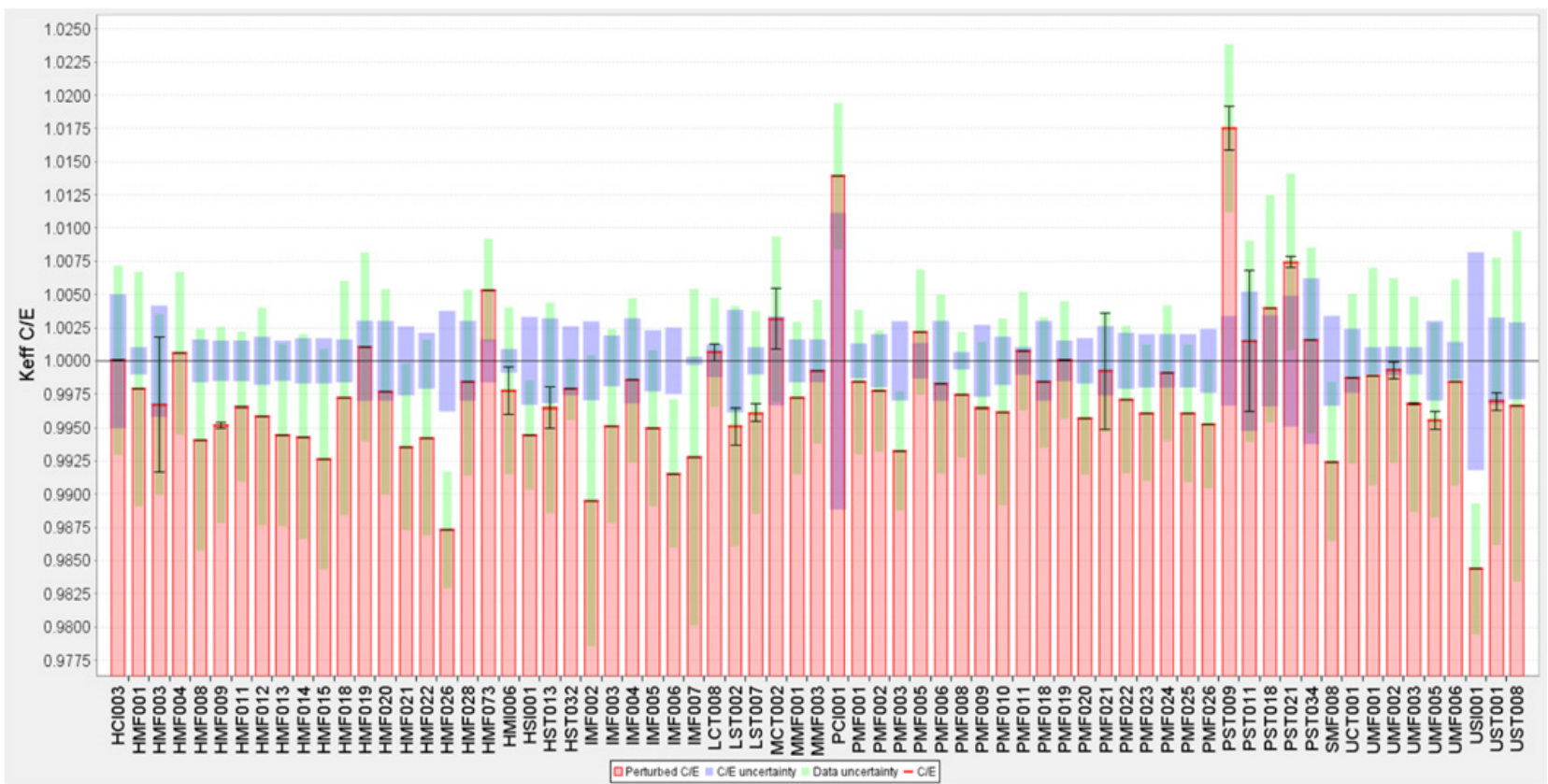

Figure 2. Output plot sorted by evaluation ID.

shown graphically in a plot generated at the bottom of the dialogue window (Fig. 3).

These are represented within any energy group structure required - either single group, equally spaced logarithmically, or more generally as supplied by the user in text file format. A number of common analytical spectrum weightings may also be applied from a fixed list: Constant / PWR / General / Maxwellian / Fission; all of which may be characterised by their $\mathrm{E}$ and Theta values as chosen by the user (default values are also given). More specificity can be obtained through use of simple X-Y text file input spectra, where either linear or logarithmic interpolation may be applied to either dimension.

NDaST is also being designed to provide a 'file upload' feature, so as to avoid the necessity to already have the desired data libraries held in a JANIS database prior to launching the software. This is primarily aimed at those users testing new evaluations, and wanting to project the effect of intermediary cross-section changes that might be suggested, to the testing suite of integral experiments.

Another option it will present is a capability to run a 'Total Monte Carlo' (TMC)-like bulk series of perturbation calculations; in an analogous way 'Total NDaST' could be used to project the effect of many (in the order of hundreds or thousands) randomly perturbed nuclear data evaluations to specified integral parameters. This could circumvent the Monte Carlo computational burden otherwise necessary. The method may be limited by the assumption of linearity in the first-order sensitivity parameters, but testing indicates satisfactory results for the selected applications. Automated calculation of the mean $\Delta k_{\text {eff }}$ (for example in the case of criticality cases) and related statistics such as standard deviation and skewness / kurtosis could also be built into the code for analysis of the resulting distribution.

\section{Energy dependence breakdown}

NDaST was previously able to display in its output the breakdown of either a perturbation (i.e., $\Delta k_{\text {eff }}$ ) or uncertainty (covariance in $k_{\text {eff }}$ ) calculation as a function of the contributing nuclide-reaction pairings (including cross-reaction covariances where included). These data are accessible via separate tabs in the 'detail popup' windows which are produced upon double-clicking the tabulated result for a given benchmark case from the main output window.

However, limitation to this degree of detail alone does not allow for a deeper analysis of which regions of the energy spectrum constitute the greatest or smallest values within the total for that nuclide-reaction. The need for a means of viewing the results as a further function of 


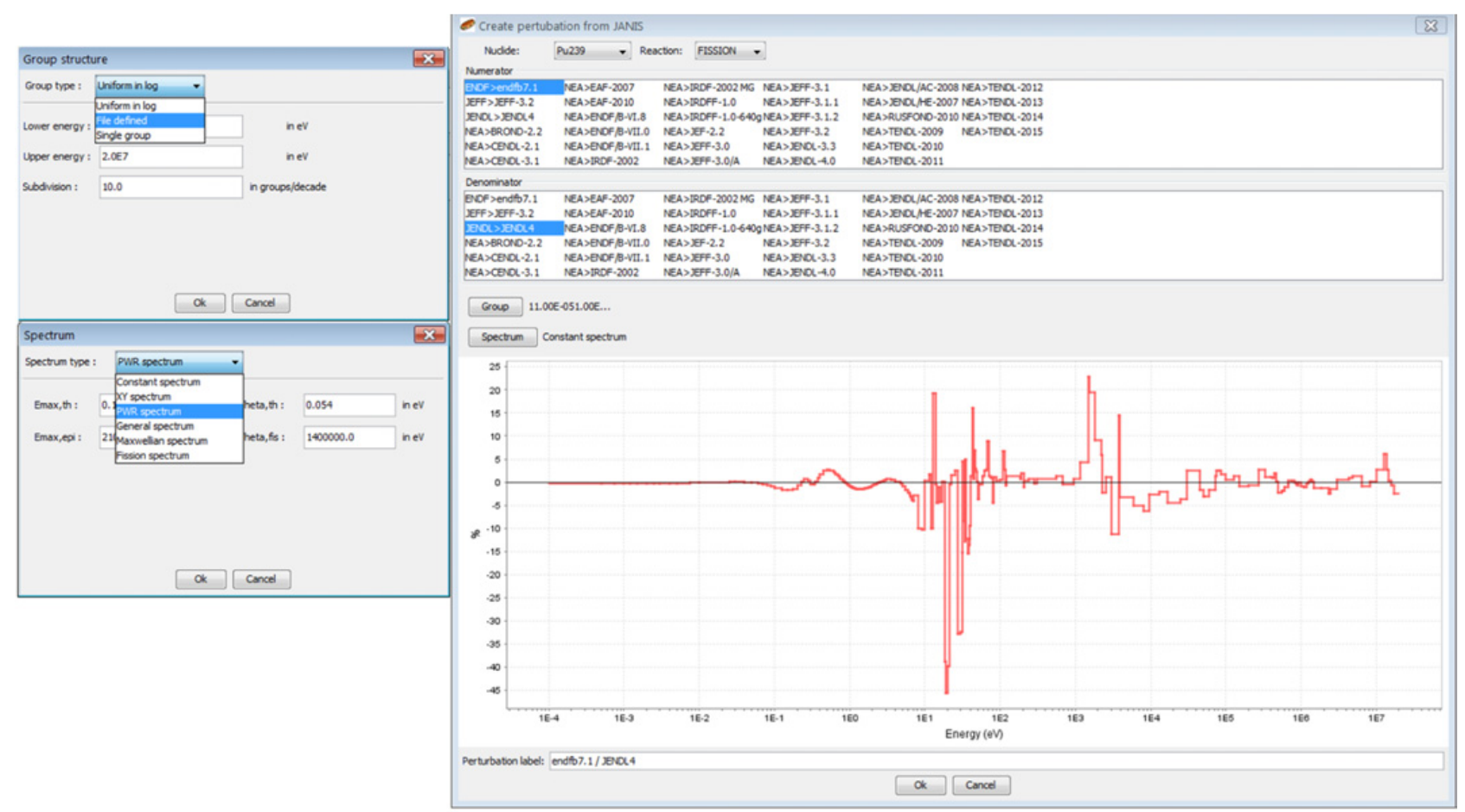

Figure 3. Automated JANIS computation dialogue.

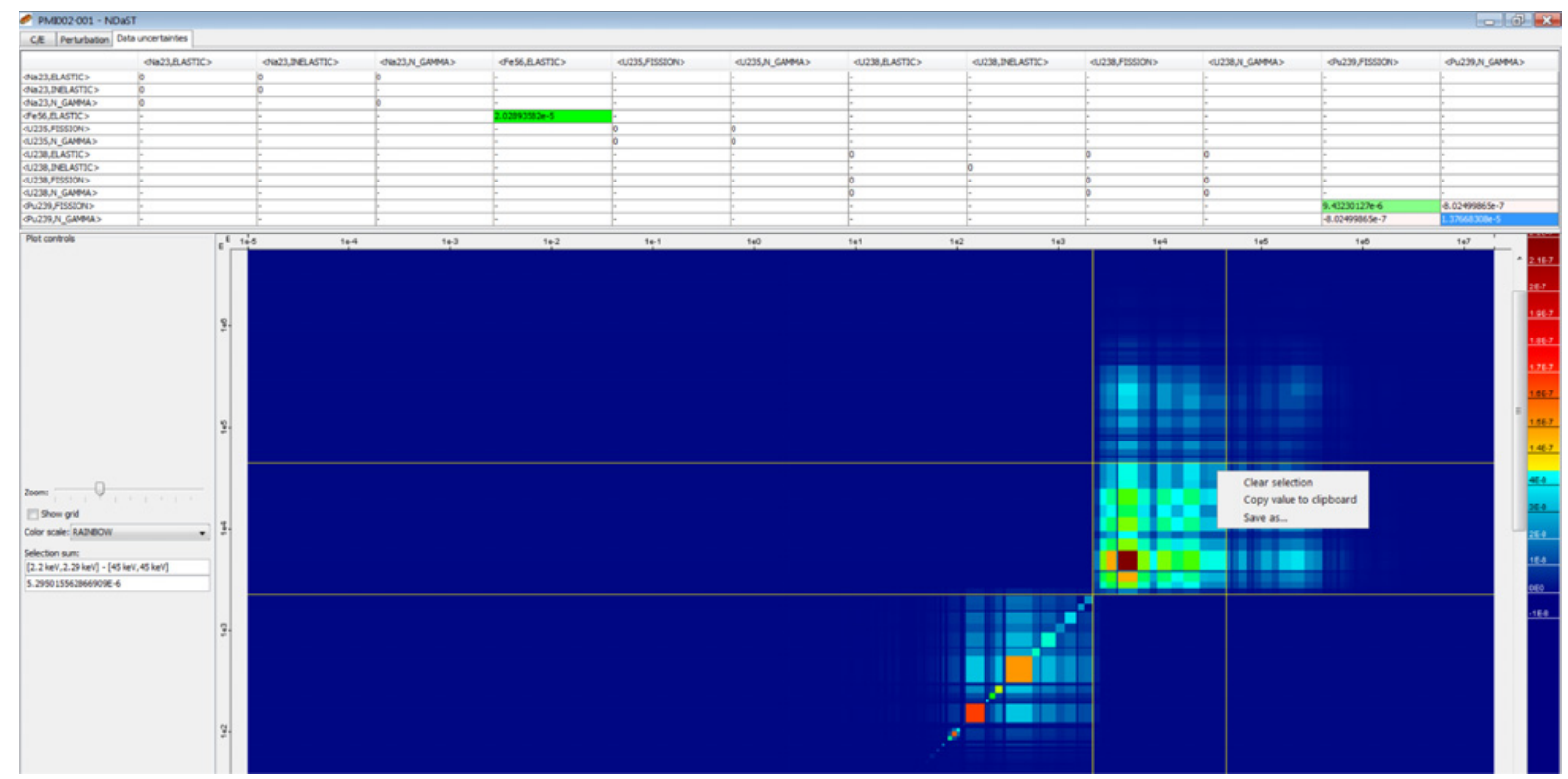

Figure 4. Energy dependence breakdown plot with 'detail popup'.

energy was clear, but the problem to overcome was the memory size of such a fine level of detail $(\sim$ thousands of energy groups, for $\sim$ hundreds of benchmarks, for $\sim$ tens of nuclide-reaction pairs; noting particularly that energy covariance matrices are large, square and often not very sparse). The manner in which one would readily and clearly display this amount of information would obviously also be challenging to effectively design.

The solution implemented was for the software to interactively relaunch the calculation specifically when clicked on by the user for a given nuclide-reaction pair. This assumes therefore that only certain pairs are of interest to the analyst at this level of resolution; for instance in testing the most important reactions, those with the greatest differential uncertainties or those related via compensating effects with one another. The energy dependence breakdowns may thus now be requested by selecting the nuclide-reaction total within the detail popup once itself opened for that benchmark case (see Fig. 4).

Furthermore, in order that totals over different ranges of energy can be quickly computed and displayed, region selection bars for choosing minimum and maximum values of the range may be used over the breakdown plot. These may be placed over regions colour-coded by their magnitude in order to permit some intuitive, visual selection of the most interesting regions to scrutinise in 


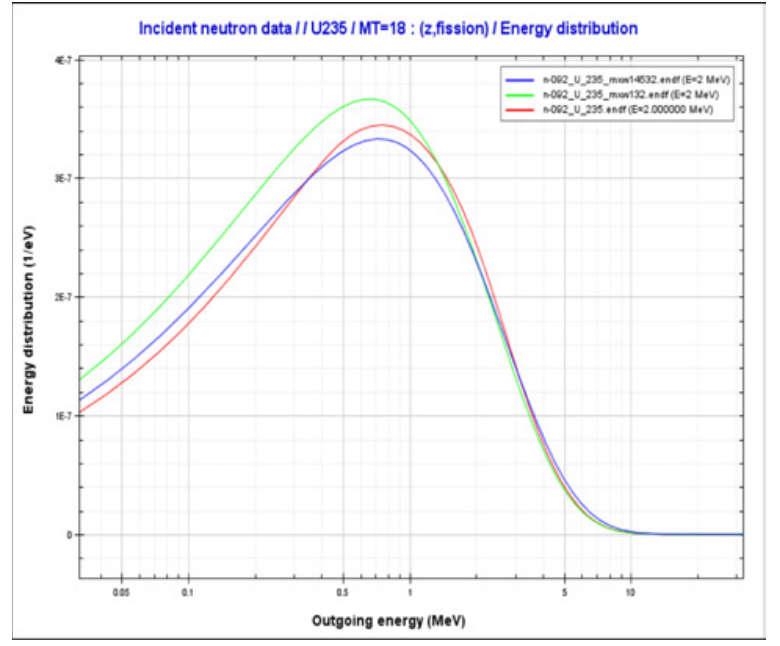

Figure 5. PFNS distributions used in perturbation tests.

added detail. The total over the selected region is displayed and values can also be output to the clipboard.

\section{Additional uncertainty capabilities}

In order for the NDaST tool to be effective for general nuclear data testing, it is desirable that it should be able to analyse as completely as possible all important nuclear data properties. In this regard, efforts have been made to provide sensitivity / uncertainty capabilities for neutron multiplicity data (nubar), prompt fission neutron spectrum (PFNS / Chi) and even angular dependence of scattering reactions, with respect to the underlying Legendre series coefficients (presently having been tested for the P1 term only).

In addition, NDaST will also incorporate the ability to compare evaluated data from nuclear data libraries with fundamental analytical expressions, such as the basic Maxwellian or Watt fission energy distributions. In order to verify perturbation techniques such as these, and to test the accuracy of sensitivity data based on a 238 multi-group energy structure, a series of simple tests were completed. The ENDF/B-VII. $1{ }^{235} \mathrm{U}$ evaluation was modified in the following three ways (see Fig. 5):

- Original is an arbitrary probability representation $(\mathrm{LF}=1)$ at 20 incident energies.

- It was changed to a Maxwellian distribution $(\mathrm{LF}=7)$ at neutron temperature of $1.32 \mathrm{MeV}$, independent of incident neutron energy.

- Also produced was another Maxwellian at $10 \%$ greater neutron temperature of $1.452 \mathrm{MeV}$.
Table 1. Comparison of direct and sensitivity based PFNS perturbations to case HEU-MET-FAST-062.

\begin{tabular}{|c|c|c|}
\hline Perturbation & $\begin{array}{c}\Delta \boldsymbol{k} \text { MCNP } \\
\text { (reference) }\end{array}$ & $\begin{array}{c}\Delta \boldsymbol{k} \\
\text { NDaST }\end{array}$ \\
\hline $\begin{array}{c}\text { ENDF/B-VII.1 evaluation } \\
\rightarrow \text { Maxwellian 1.32 MeV }\end{array}$ & $-183 \pm 21 \mathrm{pcm}$ & $-118 \mathrm{pcm}$ \\
\hline $\begin{array}{c}\text { Maxwellian } 1.32 \mathrm{MeV} \\
\rightarrow \text { Maxwellian } 1.452 \mathrm{MeV}\end{array}$ & $+988 \pm 21 \mathrm{pcm}$ & $+925 \mathrm{pcm}$ \\
\hline
\end{tabular}

These were used to test the prediction of both perturbations for HEU-MET-FAST-062 made by NDaST and via direct calculation (results are given in Table 1):

1. Using a $1 \mathrm{keV}$ linear interpolation in JANIS, then averaging back to 238 group and input to NDaST.

2. Processing modified ENDF files to make MCNP compatible ACE files (considered as reference value).

$$
\begin{gathered}
\int_{0}^{\infty} \chi(E) d E=1 \quad \chi(E)=A \sqrt{E} e^{-b E} \\
A=\frac{2 b^{3 / 2}}{\sqrt{\pi}} \quad b=\frac{1}{T} \quad\langle E\rangle=\frac{3}{2 b}
\end{gathered}
$$

The differences shown in Table 1 between MCNP and NDaST are within reasonable agreement, taking into account statistical uncertainty in the both the MCNP $k_{\text {eff }}$ calculations and the sensitivity coefficients. The extent of the non-linearity effect is also unquantified.

\section{Conclusions}

Following a testing period, new uncertainty analysis features have been implemented to the NDaST release version. This free online tool for integral benchmark testing is now available at www.oecd-nea.org/ndast/. The tool is aimed to be both efficient and comprehensive, meeting the needs of the nuclear data and benchmark testing communities.

\section{References}

[1] Dyrda, J., Hill, I., Bossant, M., Gulliford, J. \& Soppera, N., "The New OECD-NEA Nuclear Data and Sensitivity Tool (NDaST)" Proc. ANS Winter Meeting, Washington DC (2015)

[2] Hill, I., Dyrda, J., Bossant, M., Gulliford, J. \& Soppera, N., "Study on MCNP6.1 + JANIS Method to Generate P1 Sensitivity Coefficient Data, For the Purpose of Identifying ICSBEP Experiments Sensitivity to Angular Scattering" Proc. PHYSOR, Sun Valley, Idaho (2016) 